\title{
Feldionenkinetik der Wassereliminierung aus den Molekül-Ionen stereoisomerer Decalin-1,4-diole ${ }^{1)}$
}

\author{
Hans-Friedrich Grützmacher* und Günter Tolkien
}

Fakultät für Chemie der Universität Bielefeld, Universitätsstraße, D-4800 Bielefeld 1

Eingegangen am 2. Mai 1978

Die Feldionenkinetik (FIK) der Wasserabspaltung aus den Molekül-lonen der 6 stereoisomeren Decalin-1,4-diole, von 6 Stereoisomeren des 2-Methyldecalin-1,4-diols und von 3 Stereoisomeren des 9-Methyl-trans-decalin-1,4-diols wurde für Zerfallszeiten von $5 \cdot 10^{-11}$ bis $5 \cdot 10^{-9} \mathrm{~s}$ untersucht. Für die normalisierten Intensitäten $I_{\mathrm{f}}(t) / I_{\mathrm{M}}$ der Ionen $\left[\mathrm{M}-\mathrm{H}_{2} \mathrm{O}\right]^{+*}$ wurden für alle Verbindungen charakteristische FIK-Kurven erhalten. Der Kurvenverlauf wird vorwiegend durch die relative sterische Anordnung beider Hydroxygruppen bestimmt, bei Verbindungen mit 1,4cis-ständigen Hydroxygruppen auch durch die übrige Molekulargeometrie. Methylsubstituenten machen sich dagegen nicht im Kurvenverlauf bemerkbar. Durch Untersuchung spezifisch deute-

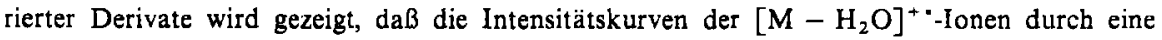
Überlagerung von mindestens 4 Eliminierungsprozessen entstehen: 1. Eine schnelle Wasserabspaltung durch 1,2-Eliminierung. 2. Eine zeitlich verzögerte 1,5-Eliminierung durch Reaktion zwischen 1,4-cis-ständigen Hydroxygruppen. Eine zeitlich verzögerte transannulare 1,4-Eliminierung bei Verbindungen mit 1,4-trans-ständigen Hydroxygruppen. 4. Eine zeitlich verzögerte Wasserabspaltung durch H-Öbertragung aus dem unsubstituierten Ring auf eine der Hydroxygruppen. Die zeitliche Verzögerung der Wasserabspaltung wird im Zusammenhang mit intramolekularen Bewegungen der Molekül-Ionen diskutiert.

\section{Field Ion Kinetics of the Water Elimination from Molecular Ions of Stereoisomeric Decalin-1,4-diols ${ }^{11}$}

The field ion kinetics (FIK) of the elimination of $\mathrm{H}_{2} \mathrm{O}$ from the molecular ions of the 6 stereoisomeric decalin-1,4-diols, of 6 stereoisomers of 2-methyldecalin-1,4-diol, and of 3 stereoisomers of 9-methyl-trans-decalin-1,4-diol has been investigated for reaction times between $5 \cdot 10^{-11}$ and $5 \cdot 10^{-9} \mathrm{~s}$. Characteristic FIK-curves of normalized intensities $I_{f}(t) / I_{M}$ of the $\left[\mathrm{M}-\mathrm{H}_{2} \mathrm{O}\right]^{+\cdot}$ ions have been obtained for all compounds. The structure of the curves is mainly determined by the relative steric orientation of both hydroxy groups, in compounds with a 1,4-cis-orientation of the hydroxy groups also by the geometry of other parts of the molecules. Methyl groups, however, have no noticeable effect on the structure of the curves. The investigation of specifically deuterated derivatives reveals, that the intensity curves of the $\left[\mathrm{M}-\mathrm{H}_{2} \mathrm{O}\right]^{+\bullet}$ ions are the result of a superposition of at least 4 elimination reactions: 1.) A rapid loss of water by 1,2-elimination. 2.) A retarded 1,5-elimination by a reaction between hydroxy groups in a 1,4-cis-position. 3.) A retarded transannular 1,4-elimination in compounds with a 1,4-trans-orientation of the hydroxy groups. 4.) A retarded loss of water by $\mathrm{H}$-transfer from the unsubstituted ring to one of the hydroxy groups. The retardation of the loss of water is discussed with respect to intramolecular movements of the molecular ions.

Das Peakmuster eines Massenspektrums, das durch Elektronenstoßionisation (EI) erzeugt und mit den üblichen Techniken aufgenommen wurde, entsteht durch die Summe der Ionen, die

(c) Verlag Chemie, GmbH, D-6940 Weinheim, 1979 
innerhalb der Aufenthaltsdauer von ca. $10^{-6} \mathrm{~s}$ in der Ionisierungskammer eines Massenspektrometers gebildet werden. Demgegenüber werden in einem durch Feldionisation (FI) erzeugten Massenspektrum wegen des großen Gradienten des elektrischen Beschleunigungsfeldes in einer FI-Ionenquelle unter den normalen Betriebsbedingungen eines doppelfokussierenden Massenspektrometers neben den Molekül-Ionen nur solche Fragment-Ionen registriert, die innerhalb von $10^{-11} \mathrm{~s}$ entstanden sind ${ }^{2)}$. In doppelfokussierenden Massenspektrometern wirkt das elektrostatische Ablenkfeld als Energiefilter, das nur Ionen mit maximaler kinetischer Energie passieren können, die innerhalb einer schmalen Ionisierungszone von einigen $10^{-8} \mathrm{~cm}$ vor dem Emitter der FI-Ionenquelle entstanden sind. Durch ein geeignetes Abstimmen des elektrischen Beschleunigungs- und Ablenkfeldes können jedoch selektiv Fragment-Ionen registriert werden, die zu einer bestimmten Zeit aus den Molekül-Ionen während des Fluges durch die FI-Ionenquelle gebildet werden. Die Zerfallszeit kann dabei innerhalb der vom jeweiligen Massenspektrometer gesetzten Grenzen von ca. $10^{-11}$ bis $10^{-8} \mathrm{~s}$ variiert werden. Diese Methode wird als FeldionenKinetik (FIK) bezeichnet ${ }^{3)}$.

Reaktionszeiten von $10^{-11}$ bis $10^{-8} \mathrm{~s}$ liegen in den Zeitbereichen, in denen auch Konformationsumwandlungen organischer Moleküle erfolgen ${ }^{4)}$. Es ist daher zu erwarten, daß schnelle Zerfallsreaktionen nicht nur von der Aktivierungsenergie, sondern auch von der molekularen Beweglichkeit der untersuchten Verbindungen beeinflußt werden.

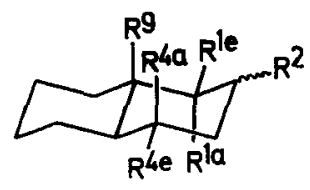

\section{Schema 1}

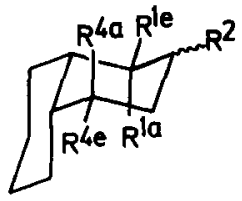

\begin{tabular}{|c|c|c|c|c|c|c|}
\hline & $\mathrm{R}^{\mathrm{le}}$ & $R^{l a}$ & $R^{4 e}$ & $R^{4 a}$ & $R^{2}$ & $R^{9}$ \\
\hline 1 & $\mathrm{OH}$ & $H$ & $\mathrm{OH}$ & $\mathrm{H}$ & $H$ & $H$ \\
\hline 2 & $H$ & $\mathrm{OH}$ & $\mathrm{H}$ & $\mathrm{OH}$ & $\mathrm{H}$ & $\mathrm{H}$ \\
\hline 3 & $\mathrm{OH}$ & $\mathrm{H}$ & H & $\mathrm{OH}$ & $\mathrm{H}$ & $\mathrm{H}$ \\
\hline$\underline{\underline{7}}$ & $\mathrm{OH}$ & $\mathrm{H}$ & $\mathrm{OH}$ & H & $\mathrm{CH}_{3}$ & $\mathrm{H}$ \\
\hline$\underline{8}$ & $\mathrm{H}$ & $\mathrm{OH}$ & H & $\mathrm{OH}$ & $\mathrm{CH}_{3}$ & $\mathrm{H}$ \\
\hline 0 & $\lceil\mathrm{OH}$ & $\mathrm{H}$ & $\mathrm{H}$ & $\mathrm{OH}$ & & \\
\hline$\underline{\underline{y}}$ & $\{H$ & $\mathrm{OH}$ & $\mathrm{OH}$ & $H$ & & $H$ \\
\hline 13 & $\mathrm{OH}$ & $H$ & $\mathrm{OH}$ & $H$ & $H$ & $\mathrm{CH}_{3}$ \\
\hline 14 & H & $\mathrm{OH}$ & $\mathrm{H}$ & $\mathrm{OH}$ & $\mathrm{H}$ & $\mathrm{CH}_{3}$ \\
\hline 15 & $\mathrm{H}$ & $\mathrm{OH}$ & $\mathrm{OH}$ & H & $H$ & $\mathrm{CH}_{3}$ \\
\hline
\end{tabular}

\begin{tabular}{c|ccccc} 
& $R^{l e}$ & $R^{l a}$ & $R^{4 e}$ & $R^{4 a}$ & $R^{2}$ \\
\hline$\underline{b}$ & $\mathrm{OH}$ & $\mathrm{H}$ & $\mathrm{OH}$ & $\mathrm{H}$ & $\mathrm{H}$ \\
$\underline{5}$ & $\mathrm{OH}$ & $\mathrm{H}$ & $\mathrm{H}$ & $\mathrm{OH}$ & $\mathrm{H}$ \\
$\underline{6}$ & $\mathrm{H}$ & $\mathrm{OH}$ & $\mathrm{OH}$ & $\mathrm{H}$ & $\mathrm{H}$ \\
$\underline{\underline{10}}$ & $\mathrm{OH}$ & $\mathrm{H}$ & $\mathrm{OH}$ & $\mathrm{H}$ & $\mathrm{CH}_{3}$ \\
$\underline{\underline{11}}$ & $\mathrm{OH}$ & $\mathrm{H}$ & $\mathrm{H}$ & $\mathrm{OH}$ & $\mathrm{CH}_{3}$ \\
$\underline{12}$ & $\mathrm{H}$ & $\mathrm{OH}$ & $\mathrm{OH}$ & $\mathrm{H}$ & $\mathrm{CH}_{3}$
\end{tabular}

In einer Reihe von Untersuchungen ist am Beispiel stereoisomerer cyclischer Diole gezeigt worden $^{5-7)}$, daß in den EI-Massenspektren eine sterische Kontrolle energetisch günstiger Eliminierungsreaktionen aus den Molekül-Ionen beobachtet werden kann. Im Fall der Diole gilt dies für eine transannulare Wasserabspaltung zwischen beiden Carbinolgruppierungen, so da 3 zwischen der relativen Orientierung der Hydroxygruppen am Ring und zwischen der Intensität der $\mathrm{M}-\mathrm{H}_{2} \mathrm{O}^{7+-}$-Ionen sowie der Deuteriumretention in diesen Ionen bei spezifisch deuterierten Diolderivaten eine übersichtliche Beziehung besteht. Am Beispiel stereoisomerer 5-tert-Butyl- 
1,3-cyclohexandiol-Derivate läßt sich aber zeigen ${ }^{8}$, $\mathrm{daB}$ nicht nur die cis-oder trans-Anordnung beider Hydroxygruppen in 1,3- oder 1,4-Stellung am Ring einen Einfluß auf die Bildung der

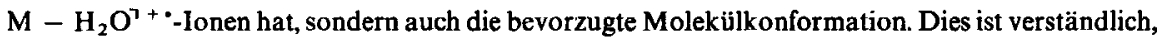
weil die intramolekularen Eliminierungen aus den Molekïl-Ionen sterisch spezielle Übergangszustände erfordern und deswegen die Reaktion auch von der Wahrscheinlichkeit abhängt, mit der ein Molekül-Ion die „reaktive Konformation“ des Übergangszustandes einnehmen kann.

Der Einfluß dieser intramolekularen Orientierungsphänomene auf schnelle Reaktionen sollte mit der FIK-Technik gut beobachtet werden können. In diesem Fall sollte die FIK-Technik auch eine neue Methode sein, um stereoisomere organische Verbindungen zu charakterisieren und zu identifizieren. Zur Prüfung dieser Überlegungen haben wir bei den Stereoisomeren 1 - 6 des Decalin-1,4-diols die Wasserabspaltung aus den MolekülIonen nach der FIK-Methode untersucht. Außerdem wurden die Stereoisomeren 7-12 des 2-Methyldecalin-1,4-diols und die Stereoisomeren 13-15 des 9-Methyl-trans-decalin1,4-diols in Analogie zu bekannten Methoden ${ }^{9)}$ hergestellt und in die Untersuchungen einbezogen. Bei den Verbindungen 7-12 handelt es sich jedoch bezüglich der sterischen Anordnung der Methylgruppe jeweils um ein Gemisch beider Stereoisomeren, das nicht aufgetrennt werden konnte. Es ist bekannt ${ }^{10)}$, daß die Methylsubstituenten dieser Verbindungen die sterische Kontrolle der Wassereliminierung in den EI-Massenspektren herabsetzen oder verdecken.

\section{Ergebnisse}

Die Meßergebnisse werden bei der FIK-Methode in der Form von Kurven der Intensität $I_{\mathrm{f}}$ des Produkt-Ions in Abhängigkeit von der kinetischen Energie der Ionen erhalten. Bei bekanntem Verlauf des Beschleunigungspotentials in der FI-Ionenquelle und bekannten Massen sowie Ladungen der Edukt- und Produkt-Ionen können daraus die Zerfallsorte in der Ionenquelle und die Lebensdauer der Edukt-Ionen bis zum Zerfall (Zerfallszeit $t$ ) berechnet ${ }^{11)}$ und Intensitäts-Zeit-Kurven $I_{\mathrm{f}}(t)$ erhalten werden. Bei den Untersuchungen dieser Arbeit wurde die kinetische Energie der Produkt-Ionen durch schrittweises Erhöhen des Feldemitter-Potentials bei konstantem Magnetfeld und elektrischem Ablenkfeld erhalten ${ }^{12}$. Diese Meßtechnik verbindet den Vorteil der einfachen Durchführung mit dem Nachteil, daß sich wegen der veränderten Potentialdifferenz zwischen Feldemitter und Gegenelektrode in der FI-Ionenquelle auch die totale Ionenausbeute ändert. Für diesen Effekt kann jedoch durch gleichzeitige Messung des TotalIonenstroms bei verschiedenen Emitter-Potentialen eine Korrektur angebracht werden.

Das für die Messungen eingesetzte Massenspektrometer Varian MAT 311 A mit kombinierter EI/FI-Ionenquelle hat für FIK-Messungen den Nachteil, daß der Bereich des Emitter-Potentials auf $+1 \mathrm{kV}$ bis $+3 \mathrm{kV}$ beschränkt ist und die für eine Feldionisation notwendige Potentialdifferenz von 6-9 kV durch Anlegen einer negativen Spannung (gegen Erdpotential) an die als Gegenelektrode verwendete Saugblende erzeugt wird. Die Ionen werden daher nach Durchfliegen der Saugblende auf ihrem Weg zu dem auf Erdpotential liegenden Austrittsspalt abgebremst und besitzen in einem bestimmten Bereich vor und hinter der Saugblende die gleiche kinetische Energie. Dieser Bereich kann somit für FIK-Messungen nicht genutzt werden. Im Vergleich zu speziellen für FIK-Messungen konstruierten Massenspektrometern ${ }^{13)}$ ist der beobachtete Zeitbereich zu langen Zerfallszeiten eingeschränkt und beträgt bei Messungen mit verschiedenen negativen Potentialen an der Gegenelektrode für einfach positiv geladene Ionen der Massen $150-200 \mathrm{u}$ ca. $5 \cdot 10^{-11}-5 \cdot 10^{-9} \mathrm{~s}$. 

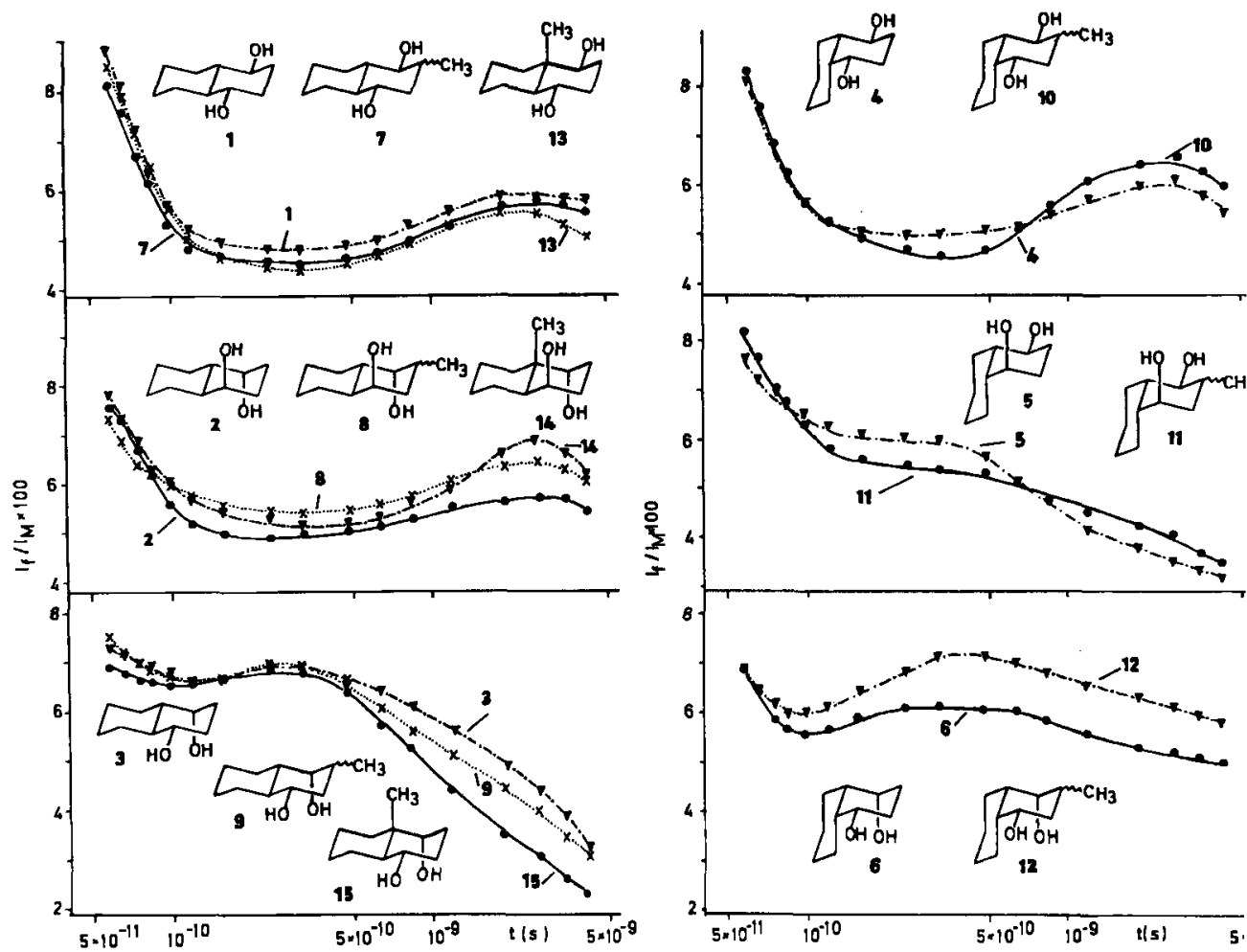

[180/78.1]

Abb. 1. Normalisierte Intensitätskurven der Ionen $\left[\mathrm{M}-\mathrm{H}_{2} \mathrm{O}\right]^{+\cdot}$ für trans- und cis-Decalin1,4-diole

Die korrigierten und auf der Intensität $I_{M}$ der Molekül-Ionen normalisierten FIKKurven $I_{\mathrm{f}}(t) / I_{\mathrm{M}}$ sind in Abb. 1 für die trans- und cis-Decalin-1,4-diole aufgeführt. Dabei wurden die Isomeren mit diaxialer, diäquatorialer und axial-äquatorialer Anordnung der Hydroxygruppen zusammen aufgetragen. Die angegebenen Kurven wurden aus jeweils 5 Einzelmessungen errechnet, sie sind bei sorgfältiger Durchführung der Messungen überraschend gut reproduzierbar, insbesondere bleibt bei allen Einzelmessungen der für die jeweilige Verbindung charakteristische Kurvenverlauf und die Lage der Maxima und Minima erhalten. Für die Umrechnung der experimentell bestimmten kinetischen Energien der Ionen in Zerfallszeiten wurde eine von Beckey et al. ${ }^{14)}$ für FI-Ionenquellen mit Drahtemittern angegebene Formel benutzt. Die Genauigkeit, mit der die Zerfallszeiten bestimmt werden können, hängt danach $u$. a. von den Fehlern bei der Bestimmung der Krümmungsradien der Emitter und der Abstände Emitter-Gegenelektrode ab. Es ist möglich, daß bei der Umrechnung der Energie- in die Zeitskala ein systematischer Fehler auftritt, der einen Faktor 2 erreichen kann. Dieser Fehler gilt jedoch in gleichem Ausmaß für alle angegebenen FIK-Kurven und verändert die in Abb. 1 für die einzelnen Stereoisomeren beobachteten Unterschiede nicht. 

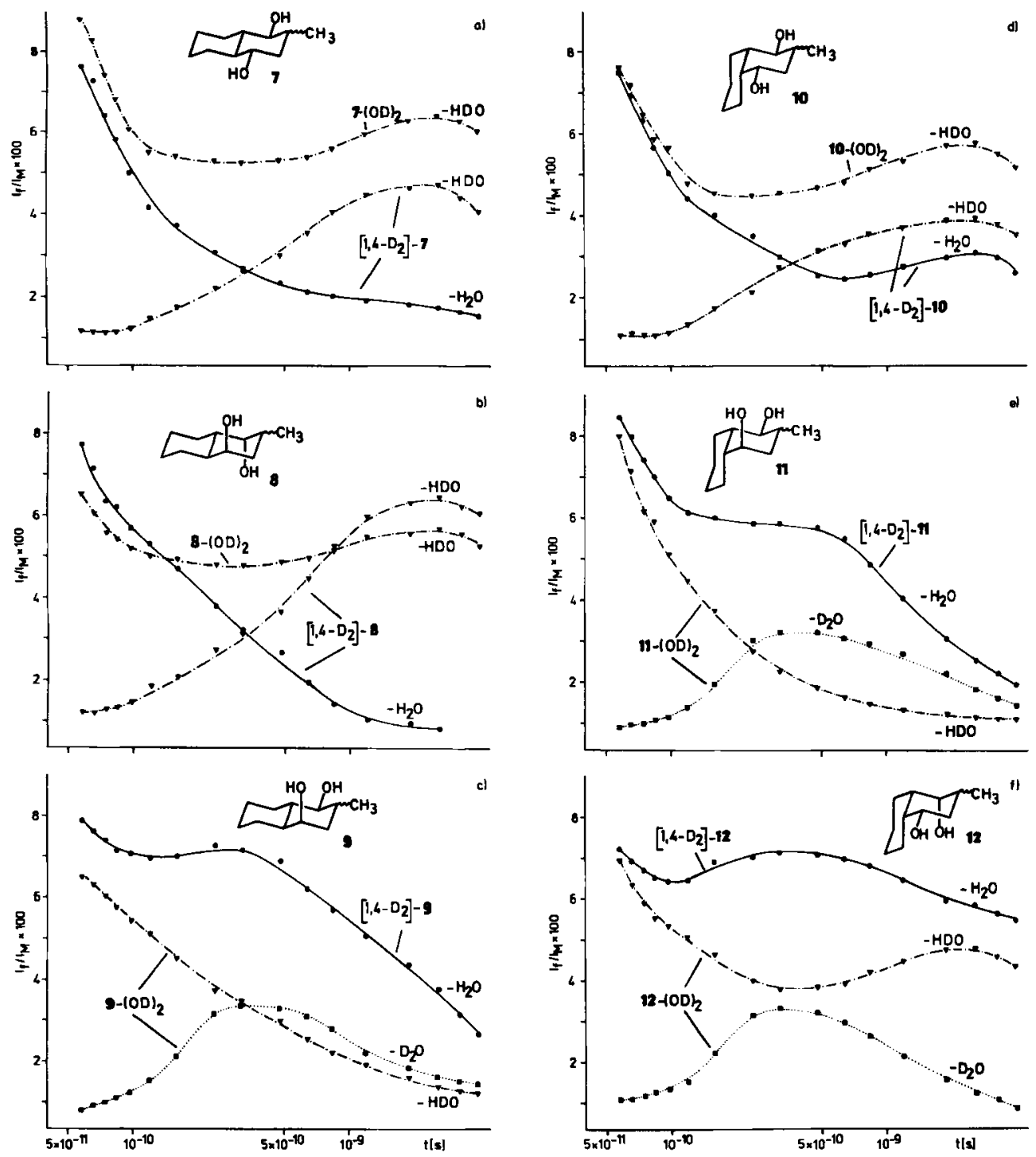

$[\overline{C 18078.2]}$

Abb. 2. Normalisierte Intensitätskurven für die Abspaltung von $\mathrm{H}_{2} \mathrm{O}, \mathrm{HDO}$ und $\mathrm{D}_{2} \mathrm{O}$ aus den Molekül-Ionen von deuterierten Derivaten der 2-Methyldecalin-1,4-diole

Um festzustellen, ob an der Wasserabspaltung aus den durch Feldionisation erzeugten Molekül-Ionen verschiedene Prozesse beteiligt sind, wurden von den Verbindungen 7-15 die in den Hydroxygruppen deuterierten Derivate 7-(OD $)_{2}-15-(O D)_{2}$ sowie von den Verbindungen 7-15 die in den Positionen 1 und 4 dideuterierten Derivate [1,4$\left.\mathrm{D}_{2}\right]-7-\left[1,4-\mathrm{D}_{2}\right]-15$ untersucht. 7-(OD) $)_{2}-15-(\mathrm{OD})_{2}$ spalten aus den Molekül-Ionen 
$\mathrm{D}_{2} \mathrm{O}$ ab, wenn eine 1,5-Eliminierung zwischen beiden OD-Gruppen abläuft. Alle anderen Prozesse führen zum Verlust von HDO. Bei den am Decalinring deuterierten Derivaten [1,4-D $\mathrm{D}_{2}$-7 bis -15 ergibt eine 1,4-Eliminierung im substituierten Ring der Decalin-1,4 diole eine Abspaltung von $\mathrm{HDO}$, alle anderen Eliminierungsmechanismen liefern $\mathrm{H}_{2} \mathrm{O}$. In Abb. 2 sind als Beispiel die FIK-Kurven der deuterierten Derivate der Stereoisomeren 7-12 des 2-Methyldecalin-1,4-diols aufgeführt.

Für 7-(OD) $)_{2}$ (Schema 1, $\left.\mathbf{R}^{1 \mathrm{e}}=\mathrm{R}^{4 \mathrm{e}}=\mathrm{OD}\right)$, 8-(OD) ${ }_{2}\left(\mathrm{R}^{1 \mathrm{a}}=\mathrm{R}^{4 \mathrm{a}}=\mathrm{OD}\right.$ ) und 10-(OD) $\left(R^{1 e}=R^{4 e}=O D\right)$ mit entweder diaxialer oder diäquatorialer Anordnung der Hydroxygruppen läßt sich über den untersuchten Bereich der Zerfallszeiten keine Abspaltung von $\mathrm{D}_{2} \mathrm{O}$ nachweisen, und die für die Eliminierung von $\mathrm{HDO}$ gemessenen FIK-Kurven stimmen gut mit den Kurven für die Wasserabspaltung aus den undeuterierten Verbindungen überein. Für 9-(OD) $)_{2}$ (Schema $1, \mathbf{R}^{1 \mathrm{e}}=\mathbf{R}^{4 \mathrm{a}}=\mathrm{OD}$ bzw. $\mathbf{R}^{1 \mathrm{a}}=\mathbf{R}^{4 \mathrm{e}}=\mathrm{OD}$ ), 11-(OD) $)_{2}$ $\left(R^{1 e}=R^{4 a}=O D\right)$ und $12-(O D)_{2}\left(R^{1 a}=R^{4 e}=O D\right)$ mit einer axialen und einer äquatorialen OD-Gruppe werden dagegen FIK-Kurven sowohl für die Eliminierung von $\mathrm{D}_{2} \mathrm{O}$ als auch $\mathrm{HDO}$ gefunden. Dabei ist auffällig, daß die normalisierten Intensitätskurven für $\left[\mathrm{M}-\mathrm{D}_{2} \mathrm{O}\right]^{+}$bei kurzen Zerfallszeiten mit kleinen Intensitätswerten ein-

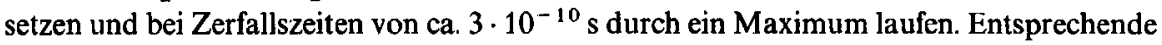
Resultate wurden auch bei der Untersuchung der übrigen Verbindungen mit axialäquatorialer Anordnung der OD-Gruppen erhalten.

Bei den am Decalinring deuterierten Verbindungen werden für [1,4- $\left.\mathrm{D}_{2}\right]-9$ (Schema 1, $\left.\mathrm{R}^{1 \mathrm{a}}=\mathrm{R}^{4 \mathrm{e}}=\mathrm{D}\right),\left[1,4-\mathrm{D}_{2}\right]-11\left(\mathrm{R}^{1 \mathrm{a}}=\mathrm{R}^{4 \mathrm{e}}=\mathrm{D}\right)$ und $\left[1,4-\mathrm{D}_{2}\right]-12\left(\mathrm{R}^{1 \mathrm{e}}=\mathrm{R}^{4 \mathrm{a}}=\mathrm{D}\right)$ ebenso wie für alle anderen am Ring deuterierten Derivate mit axial-äquatorialer Stellung der $\mathrm{OH}$-Gruppen nur Abspaltungen von $\mathrm{H}_{2} \mathrm{O}$ beobachtet, wobei die FIK-Kurven wiederum gut mit denen der undeuterierten Verbindungen übereinstimmen und somit einen Eindruck von der Reproduzierbarkeit der FIK-Messungen vermitteln. Die Verbindungen mit diaxialer oder diäquatorialer Anordnung der Hydroxygruppen, [1,4-D $\left.{ }_{2}\right]-7$ (Schema 1, $\left.\mathbf{R}^{1 \mathrm{a}}=\mathbf{R}^{4 \mathrm{a}}=\mathrm{D}\right),\left[1,4-\mathrm{D}_{2}\right]-8\left(\mathbf{R}^{1 \mathbf{e}}=\mathbf{R}^{4 \mathbf{e}}=\mathrm{D}\right)$ und $\left[1,4-\mathrm{D}_{2}\right]-10\left(\mathbf{R}^{1 \mathbf{a}}=\mathbf{R}^{4 \mathbf{a}}=\mathrm{D}\right)$, liefern dagegen FIK-Kurven sowohl fuir die Eliminierung von $\mathrm{H}_{2} \mathrm{O}$ als auch HDO. Dabei ist die Abspaltung von HDO offensichtlich wieder ein zeitlich verzögerter Prozeß, der bei relativ langen Zerfallszeiten von ca. $3 \cdot 10^{-9} \mathrm{~s}$ ein Maximum in den normalisierten Intensitätskurven liefert. Entsprechende FIK-Kurven wurden auch für $\left[1,4-\mathrm{D}_{2}\right]-13 \mathrm{mit}$ diäquatorialer und [1,4-D $\left.\mathrm{D}_{2}\right]-14$ mit diaxialer Stellung der Hydroxygruppen erhalten, wobei das Maximum in den normalisierten Intensitätskurven wiederum bei Zerfallszeiten von ca. $4 \cdot 10^{-9} \mathrm{~s}$ liegt.

\section{Diskussion}

Die Resultate dieser FIK-Untersuchungen sind in mehrfacher Hinsicht von Interesse. Die in Abb. 1 wiedergegebenen FIK-Kurven für die Wassereliminierung zeigen sowohl in der Reihe der Decalin-1,4-diole 1-6, der 2-Methyldecalin-1,4-diole 7-12 und der 9-Methyldecalin-1,4-diole 13-15 für jedes Stereoisomere einen charakteristischen Verlauf. Die Zusammenstellung der Kurven in Abb. 1 macht dabei deutlich, daß die Anwesenheit und Stellung eines Methylsubstituenten innerhalb der Reproduzierbarkeit der Methode keinen Einfluß auf den Intensitätsverlauf der $\left[\mathrm{M}-\mathrm{H}_{2} \mathrm{O}\right]^{+}-$-Ionen hat. Dies ist ein Unterschied zu den EI-Massenspektren der gleichen Verbindungen ${ }^{10}$, bei denen die 
Intensitäten und die Intensitätsunterschiede der Ionen $\left[\mathrm{M}-\mathrm{H}_{2} \mathrm{O}\right]^{+}$für die Stereoisomeren durch zusätzliche Methylgruppen im Molekül merklich beeinflußt werden. Dieser Unterschied läßt sich durch die zum Vergleich mit dem Elektronenstoß schonende Ionisierung der Moleküle bei der Feldionisation erklären, die bevorzugt Molekül-Ionen mit geringer Anregungsenergie liefert.

Während bei den hoch angeregten Molekül-Ionen der EI-Massenspektren neben und vor der Wasserabspaltung Isomerisierungen und Spaltungsreaktionen durch Bruch von $\mathrm{C}-\mathrm{H}-$ und $\mathrm{C}-\mathrm{C}$-Bindungen eintreten, deren Ausmaß durch Substituenten am Decalinring beeinflußt wird, erfolgen in den schwach angeregten Molekül-Ionen nach Feldionisation offenbar keine Bindungsspaltungen im Kohlenwasserstoffgerüst. Diese Annahme wird gestützt durch die FI-Massenspektren der Decalin-1,4-diole und Methyldecalin-1,4-diole, die neben den Peaks der Molekül-Ionen und $\left[\mathrm{M}-\mathrm{H}_{2} \mathrm{O}\right]^{+\bullet}$-Ionen keine intensiven Signale enthalten. Man kann daraus den Schluß ziehen, daß mit den FI-Massenspektren und den FIK-Kurven die Reaktionen von ,intakten“ MolekülIonen untersucht werden, deren Konstitution weitgehend der von neutralen Molekülen entspricht. Dies zeigt sich auch in dem unterschiedlichen Verhalten der deuterierten Derivate der Decalindiole, das eine Isomerisierung in den Molekül-Ionen durch $\mathbf{H}$ bzw. D-Wanderungen vor der Weiterreaktion ausschließt.

Die Struktur der Kurven in Abb. 1 wird in erster Linie von der relativen sterischen Anordnung der Hydroxygruppen und erst nachgeordnet von der Geometrie des Decalingerüstes bestimmt. So besitzen die Verbindungen 1, 2, 4, 7, 8, 10, 13 und 14 mit diaxialer oder diäquatorialer Stellung der Hydroxygruppen, die einer 1,4-trans-Anordnung am Cyclohexanring entspricht, Intensitätskurven für die $\left[\mathrm{M}-\mathrm{H}_{2} \mathrm{O}\right]^{+\bullet}$-Ionen mit einem Minimum bei Zerfallszeiten von $10^{-10}-10^{-9} \mathrm{~s}$. An diesem typischen Kurvenverlauf kann daher das Vorhandensein einer 1,4-trans-Diolstruktur gut erkannt werden, allerdins ist es schwer, zwischen diaxialer und diäquatorialer Anordnung der Hydroxygruppen und einer cis- oder trans-Verknüpfung der Ringe im Decalin zu unterscheiden. Bei den übrigen Verbindungen mit axial-äquatorialer Stellung der Hydroxygruppen, entsprechend einer 1,4-cis-Anordnung dieser Gruppen am Cyclohexanring, macht sich jedoch der Einfluß der Geometrie des Decalingerüstes deutlicher bemerkbar. Dies hat zur Folge, daß 3, 9 und 15, 5 und 11 sowie 6 und 12 mit gleicher Molekülgeometrie auch jeweils untereinander sehr ähnliche Intensitätskurven der $\left[\mathrm{M}-\mathrm{H}_{2} \mathrm{O}\right]^{+\bullet}$-Ionen liefern und voneinander durch den charakteristischen Kurvenverlauf unterschieden werden können. Dies ist ein vielversprechendes Ergebnis für eine analytische Anwendung der FIK-Methode auf die Identifizierung von Stereoisomeren.

Der charakteristische Verlauf der Kurven für die normalisierten Intensitäten der Ionen $\left[\mathrm{M}-\mathrm{H}_{2} \mathrm{O}\right]^{+\cdot}$ ist auch im Hinblick auf die Mechanismen der Wassereliminierung aus den Molekül-Ionen cyclischer Diole von Interesse. Ein ungleichförmiger Verlauf der FIK-Kurven mit Minima und Maxima ist ein Hinweis, daß die Produkt-Ionen durch mehrere Zerfallsprozesse entstehen, die zu verschiedenen Zerfallszeiten eine maximale Zerfallsrate erreichen.

Bei einer Normalisierung auf die Intensität der Molekül-Ionen $I_{\mathrm{M}}$ besteht zwischen der normalisierten zeitabhängigen Geschwindigkeitskonstanten $k(t)$ des beobachteten Prozesses, der Zerfallsrate $\mathrm{d} I_{\mathrm{f}} / \mathrm{d} t$ und der Intensität der Produkt-Ionen $I_{\mathrm{f}}$ zur Zerfallszeit $t$ folgende Beziehung ${ }^{15)}$ : 


$$
\begin{aligned}
\mathrm{d} I_{\mathrm{f}} / \mathrm{d} t & =k(t) I_{\mathrm{M}}=I_{\mathrm{f}}(t) / \Delta t \\
\frac{I_{\mathrm{f}}(t)}{I_{\mathrm{M}}} & =k(t) \Delta t
\end{aligned}
$$

$\Delta t$ ist die Zeitauflösung des Massenspektrometers, die von den Massen der Molekülund Produkt-Ionen sowie von der Zerfallszeit abhängt. Da die Massen der MolekülIonen bzw. der Ionen $\left[\mathrm{M}-\mathrm{H}_{2} \mathrm{O}\right]^{+\bullet}$ bei den untersuchten stereoisomeren Verbindungen gleich oder nur wenig unterschieden sind, trifft dies auch für $\Delta t$ bei allen Messungen zu. Die normalisierten Intensitätskurven $I_{f}(t) / I_{M}$ in den Abbildungen spiegeln daher den Verlauf der normalisierten Geschwindigkeitskonstanten $k(t)$ wider.

$k(t)$ ist eine makroskopische experimentelle Größe. Die mikroskopische Geschwindigkeitskonstante $k(E)$ für die unimolekulare Reaktion eines Molekül-Ions ist nach der Quasi-Gleichgewichts-Theorie (QET) der Massenspektren ${ }^{16)}$ eine Funktion der Anregungsenergie $E$ des Molekül-Ions, der Aktivierungsenergie $E_{\mathrm{o}}$ des Prozesses und der relativen Zustandsdichte des aktivierten Komplexes im Bereich $E-E_{0}$. Bei der Feldionisation werden wie bei der Ionisation durch Elektronenstoß nicht Molekül-Ionen mit einer definierten Anregungsenergie $E$ erzeugt, sondern solche mit einer Verteilung von $E$ zwischen 0 und einigen $\mathrm{eV}^{17)}$. Dem entspricht eine Verteilung der Geschwindigkeitskonstanten $k(E)$ für die Zerfallsprozesse. Bei einem Zerfall des Molekül-Ions durch direkten Bindungsbruch mit einem ,lockeren“ aktivierten Komplex ${ }^{18)}$ wird die maximale Zerfallsgeschwindigkeit von der Schwingungsfrequenz der gespaltenen Bindung bestimmt, d. h. von der Geschwindigkeit, mit der sich beide Teile des Molekül-Ions voneinander fortbewegen können. Die sich daraus ergebenden kürzesten Zerfallszeiten von ca. $10^{-14}-10^{-13} \mathrm{~s}$ liegen außerhalb des Meßbereichs der FIK-Methode. Bei den experimentell zugänglichen längeren Zerfallszeiten von $10^{-11} \mathrm{~s}$ werden daher für diese Reaktionen in den Kurven für die normalisierten Intensitäten $I_{f}(t) / I_{M}$ oder der Geschwindigkeitskonstanten $k(t)$ bereits hohe Werte erwartet, die den schnellen Reaktionen energiereicher Molekül-Ionen entsprechen. Zu langen Zerfallszeiten nehmen diese Werte entsprechend den langsameren Reaktionen der energieärmeren Molekül-Ionen gleichförmig ab. Derartige FIK-Kurven wurden wiederholt beobachtet ${ }^{19)}$. Die maximale Geschwindigkeit des Zerfalls eines Molekül-Ions durch Umlagerungen oder Eliminierungen kann jedoch erheblich kleiner sein als beim direkten Bindungsbruch, weil „feste“ Übergangszustände mit sterisch spezifischen Konformationen durchlaufen werden. Für den Zerfall eines linearen Keton-Ions durch eine McLafferty-Umlagerung wurde abgeschätzt, daß der Zeitraum bis zum zufälligen Erreichen des sechsgliedrigen Übergangszustandes durch Bindungsrotationen $10^{-9}-10^{-10} \mathrm{~s}$ beträgt ${ }^{20}$. In den FIK-Kurven für diese Zerfallsprozesse werden daher für kurze Zerfallszeiten von $10^{-11} \mathrm{~s}$ trotz der ausreichenden Anregungsenergie der Molekül-Ionen kleine Werte für $I_{\mathrm{f}}(t) / I_{\mathrm{M}}$ bzw. $k(t)$ erwartet, weil die Wahrscheinlichkeit zum Erreichen der reaktiven Konformation bei kurzen Zeiten gering ist. Mit dem Ansteigen dieser Wahrscheinlichkeit nimmt zu längeren Zerfallszeiten auch $I_{\mathrm{f}}(t) / I_{\mathrm{M}}$ oder $k(t)$ zunächst $\mathrm{zu}$, um anschließend zu langen Reaktionszeiten entsprechend dem normalen Einfluß der Verteilung der Anregungsenergie in den MolekülIonen abzuklingen. Diese Erwartung wird durch den Nachweis einer zeitlichen Verzögerung von Umlagerungsreaktionen in den FIK-Kurven erfïllt ${ }^{20}$ ). 
Die zeitliche Verzögerung des Prozesses und die Lage des Maximums in den FIKKurven wird dabei offensichtlich von den geometrischen Ansprüchen des Übergangszustandes bestimmt. So haben Derrick, Falick und Burlingame nachgewiesen ${ }^{21)}$, daß bei der Abspaltung von $\mathrm{H}_{2} \mathrm{O}$ aus den Molekül-Ionen von Hexanol ein fünfgliedriger Übergangszustand bei kurzen Zerfallszeiten relativ $\mathrm{zu}$ dem "normalen" sechsgliedrigen Übergangszustand begünstigt wird. Ebenso zeigen die Untersuchungen zur Wasserabspaltung aus Cyclohexanol-Ionen von Derrick, Holmes und Morgan ${ }^{22)}$, daß bei kurzen Zerfallszeiten eine 1,2-Eliminierung überwiegt und die aus den EI-Massenspektren bekannte transannulare 1,4-Abspaltung erst bei längeren Zerfallszeiten zum Zuge kommt. Eine 1,3-Eliminierung aus den Cyclohexanol-Ionen, die in den EI-Spektren ebenfalls auftritt und der wahrscheinlich eine Aufspaltung des Cyclohexanringes vorausgeht ${ }^{23}$, wird dagegen nicht beobachtet.

Die Untersuchung der deuterierten Derivate der Decalin-1,4-diole zeigt, daß die in Abb. 1 wiedergegebenen FIK-Kurven für die Ionen $\left[\mathrm{M}-\mathrm{H}_{2} \mathrm{O}\right]^{+\bullet}$ bei diesen Verbindungen durch die Überlagerung eines schnellen, zeitlich nicht verzögerten Prozesses mit mindestens drei zeitlich verzögerten Eliminierungsreaktionen entstehen.

Der schnelle Eliminierungsproze $B$ gibt sich in $A b b .2$ bei $\left[1,4-D_{2}\right]-7$ und -8 durch die Kurven für die Abspaltung von $\mathrm{H}_{2} \mathrm{O}$, bei 9-(OD) $)_{2}$ und 11-(OD) $)_{2}$ durch die Kurven für die Abspaltung von HDO zu erkennen. Die Kurven setzen bei kurzen Zerfallszeiten mit hohen Intensitätswerten ein und fallen zu längeren Zerfallszeiten gleichförmig schnell ab. Der Übergangszustand dieser Eliminierung muß daher sterisch leicht erreicht werden können und von der Ausgangskonformation der Molekül-Ionen und Konformationsänderungen wenig beeinflußt werden.

Bei der FIK-Untersuchung eines 2,2,3,3,9,10-Hexadeuterio-trans-decalin-1,4-diols (mit diäquatorialer Stellung der Hydroxygruppen) tritt dieser Prozeß als Abspaltung von HDO auf, so daß an ihm nur D-Atome des hydroxylsubstituierten Ringes beteiligt sind, entsprechend einer 1,2- oder 1,3-Wassereliminierung. Die Analogie zum Mechanismus der Wassereliminierung aus Cyclohexanol-Ionen ${ }^{22)}$ spricht dabei mit hoher Wahrscheinlichkeit für eine 1,2-Eliminierung (Schema 2).

\section{$\underline{\text { Schema } 2}$}

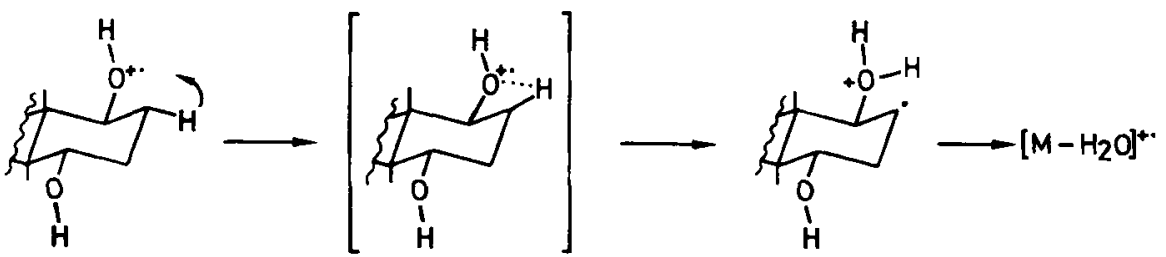

Für diese Reaktion wird eine vergleichsweise hohe Aktivierungsenergie angenommen ${ }^{22)}$, so daß sie nur in energiereichen Molekül-Ionen eintritt und zu längeren Reaktionszeiten schnell abklingt. Als Ursache für die große Aktivierungsenergie wird ein großer Abstand von 2.3-2.5 $\AA$ der Atommittelpunkte des Sauerstoffatoms der Hydroxygruppe und eines Wasserstoffatoms an benachbarten Kohlenstoffatomen vermutet, so daB eine erhebliche Dehnung der Bindungen bei der Wasserstoffübertragung im Übergangśzustand eintreten muß. 
Zum Erreichen des (viergliedrigen) cyclischen Übergangszustandes ist aber keine komplizierte Molekülbewegung notwendig, und die sterische Ausgangslage dafür ist weitgehend unabhängig von der Konfiguration und Konformation des Molekül-Ions. Wie Abb. 3 zeigt, fallen daher die FIK-Kurven für diesen schnellen Prozeß bei allen Verbindungen in einen relativ schmalen Bereich.

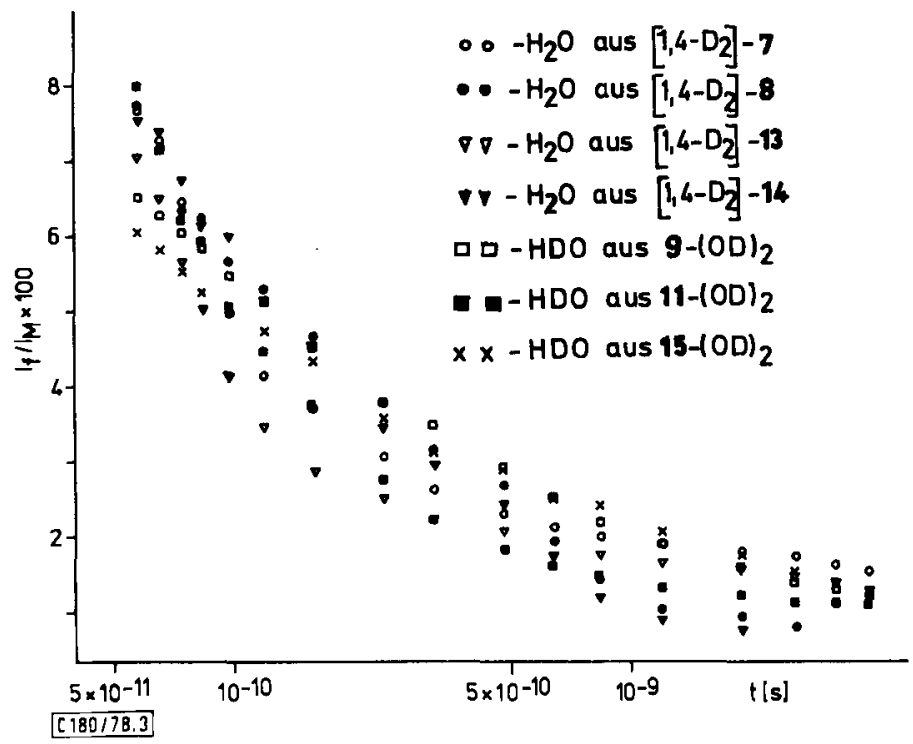

Abb. 3. Normalisierte Intensitätskurven für die Abspaltung von $\mathrm{H}_{2} \mathrm{O}$ aus den Molekül-Ionen durch 1,2-Eliminierung

Dies ist insofern bemerkenswert, als bei längeren Zerfallszeiten in den verschiedenen Verbindungen unterschiedliche Reaktionen mit der schnellen Wasserabspaltung konkurrieren. Dies macht sich in Abb. 3 durch Verschiebungen der einzelnen Kurven innerhalb der Zone auch bemerkbar, ändert aber den Kurvenverlauf nicht entscheidend. Man kann daher annehmen, daß der Verlauf der FIK-Kurven vornehmlich durch die Eigenschaften des untersuchten Prozesses und weniger durch die Konkurrenz mit anderen Zerfallsreaktionen bestimmt wird.

\section{Schema 3}

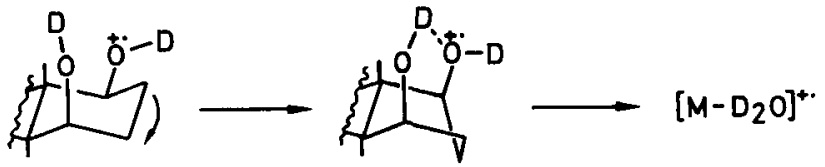

Ein zweiter Mechanismus für die Abspaltung von $\mathrm{H}_{2} \mathrm{O}$ aus den Molekül-Ionen, die zeitlich verzögert erfolgt, ist die 1,5-Eliminierung durch Reaktion zwischen 1,4-ständigen Hydroxygruppen. Diese Reaktion liefert in den $\mathrm{OD}_{2}$-Derivaten die Ionen $\left[\mathrm{M}-\mathrm{D}_{2} \mathrm{O}\right]^{+\bullet}$ 
und kann aus sterischen Gründen in intakten Molekül-Ionen nur bei 1,4-cis-Stellung der Substituenten eintreten; wobei jedoch mindestens der substituierte Ring in eine Boot- oder Twistkonformation übergeführt werden muß (Schema 3), damit ein Abstand der Kernmittelpunkte des H-Atoms der einen Hydroxygruppe und des O-Atoms der anderen Gruppe von $<2 \AA$ erreicht werden kann.

Beim Ionisationsproze $\beta$ werden offensichtlich kaum Molekül-Ionen erzeugt, die in dieser reaktiven Konformation vorliegen, denn die normalisierten Intensitäten der Ionen $\left[\mathrm{M}-\mathrm{D}_{2} \mathrm{O}\right]^{+*}$ sind für Zerfallzeiten $<10^{-10} \mathrm{~s}$ bei 9-(OD) $)_{2}, 11-(\mathrm{OD})_{2}$ und 12-(OD) gering (Abb. 2). Erst nach dieser Zeit, welche die Molekül-Ionen offenbar für Konformationsänderungen benötigen, steigt die Intensität der $\left[M-\mathrm{D}_{2} \mathrm{O}\right]^{+\cdot}$-Ionen steil an, um nach einem Maximum bei ca. $3 \cdot 10^{-10} \mathrm{~s}$ in der üblichen Weise abzusinken. Im Bereich des Maximums und bei längeren Reaktionszeiten ist die Intensität der durch die 1,5Eliminierung gebildeten Ionen größer als die der durch die 1,2-Eliminierung entstandenen. Die 1,5-Abspaltung sollte demnach durch eine kleinere Aktivierungsenergie in der Konkurrenz zur 1,2-Eliminierung begïnstigt sein.

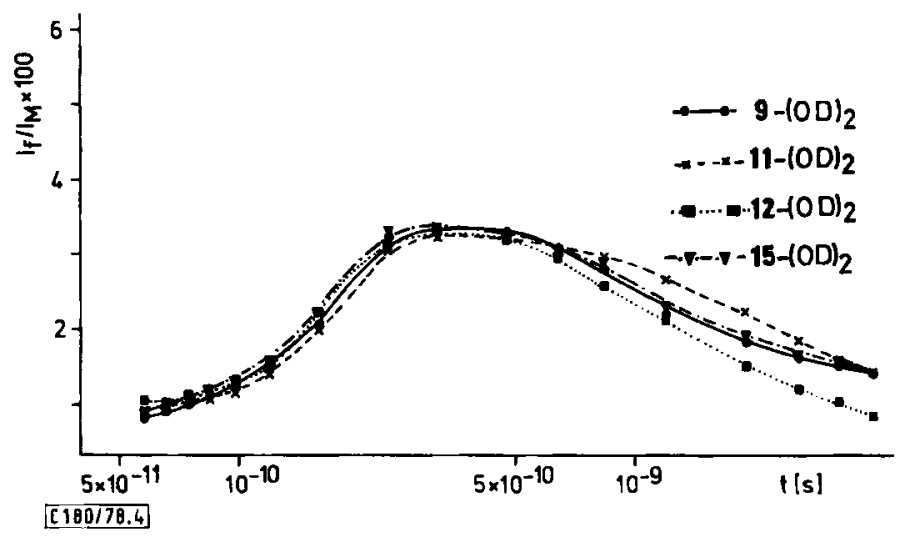

Abb. 4. Normalisierte Intensitätskurven für die Abspaltung von $\mathrm{D}_{2} \mathrm{O}$ durch 1,5-Eliminierung

Die Zusammenstellung der FIK-Kurven für die Ionen $\left[\mathrm{M}-\mathrm{D}_{2} \mathrm{O}\right]^{+}$der Decalin1,4-diole, in denen eine 1,5-Eliminierung beobachtet wird, ergibt eine ausgezeichnete Übereinstimmung (Abb. 4), obwohl die Verbindungen sich durch die cis/trans-Verknüpfung des Dekalingerüstes und durch die Stellung der Methylgruppe unterscheiden. Auch in diesem Fall wird die zeitliche Änderung der normalisierten Geschwindigkeitskonstanten $k(\mathrm{t})$ durch die spezifischen Eigenschaften des Prozesses und des zugehörenden Übergangszustandes bestimmt.

Ein weiterer Mechanismus für eine zeitlich verzögerte Wasserabspaltung wird durch

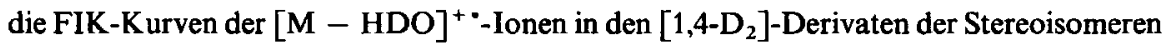
mit diaxialer oder diäquatorialer Anordnung der Hydroxygruppen angezeigt (Abb. 2). Bei dieser Reaktion handelt es sich um die aus den EI-Massenspektren cyclischer Diole ${ }^{6,7)}$ bekannte transannulare 1,4-Eliminierung. Sie ist bei einer trans-Stellung der beiden Hydroxygruppen in den cyclischen Molekül-Ionen energetisch begünstigt, weil ein $\mathrm{H}$ - 
Atom unter Bruch einer schwachen $\mathrm{C}-\mathrm{H}$-Bindung in einer Carbinolgruppe ${ }^{24)}$ auf die austretende OH-Gruppe übertragen werden kann (Schema 4).

\section{Schema 4}
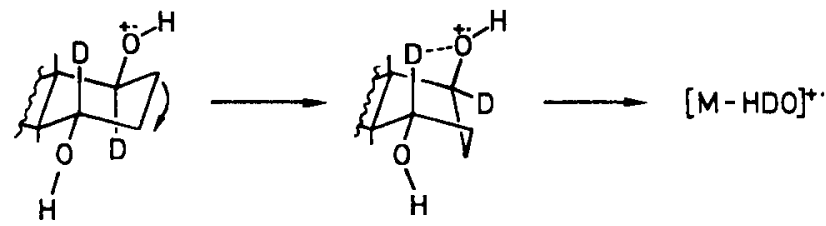

Es überrascht daher, daß trotz der geringen Aktivierungsenergie dieser Eliminierung die Zerfallsrate bei Zèiten $<10^{-10} \mathrm{~s}$ klein bleibt und die normalisierten Intensitäten der so gebildeten Ionen erst bei Reaktionszeiten von $3 \cdot 10^{-9} \mathrm{~s}$ ein Maximum erreichen. Diese sehr deutliche zeitliche Verzögerung muß ihre Ursache in einem sterisch anspruchsvollen Ubergangszustand haben, der von den Molekül-Ionen nur durch eine komplizierte Bewegung erreicht werden kann.

Es wurde bisher angenommen ${ }^{7,10)}$, daß die Übertragung eines H-Atoms aus der 4-Stellung über den Ring hinweg auf eine Hydroxygruppe im Decalingerüst ebenso wie die oben besprochene 1,5-Eliminierung nur eine Umwandlung des hydroxylierten Ringes in die Boot- oder Twistkonformation erfordert. Dies kann allein jedoch noch nicht ausreichend sein, denn beide Prozesse sollten dann bei vergleichbaren Reaktionszeiten ihre maximale Zerfallsrate erreichen und die transannulare 1,4-Eliminierung wegen der kleineren Aktivierungsenergie deutlich überwiegen. Ein direkter Vergleich beider Eliminierungsreaktionen in einem Molekül-Ion wurde bisher nicht durchgeführt. Die Verschiebung des Intensitätsmaximums der transannularen 1,4-Abspaltung um einen Faktor 10 auf der Zeitskala gegenüber der 1,5-Eliminierung ist jedoch $\mathrm{zu}$ deutlich, als daß eine Kontrolle beider Reaktionen durch gleiche intramolekulare Bewegungsabläufe wahrscheinlich ist.

Dreiding-Modelle zeigen, daß in den Decalin-1,4-diolen mit einer 1,4-trans-Stellung der Hydroxygruppen am substituierten Sechsring auch nach Umklappen dieses Ringes in die Twistform, die durch die Ringverknüpfung im Decalinsystem bedingt wird, der Abstand des O-Atoms der Hydroxygruppe und des H-Atoms in 4-Stellung mit ca. $3 \AA$ relativ groß ist. Eine Verkürzung des Abstandes zwischen den Mittelpunkten dieser Atome auf ca. $2 \AA$ oder weniger, wie er für die 1,5-Eliminierung bereits in der einfachen Twistkonformation möglich ist, kann nur nach Umklappen auch des zweiten Ringes in die Twistkonformation oder durch geeignete Deformationsschwingungen erreicht werden. Diese Betrachtung spricht dafür, daß eine intramolekulare Wasserstoffübertragung in Molekül-Ionen nur möglich ist, wenn ein Abstand zwischen den beteiligten Atomen von $\leqslant 2 \AA$ eingestellt wird, und daß ein derartiger Übergangszustand der transannularen 1,4-Abspaltung von $\mathrm{H}_{2} \mathrm{O}$ bei den Decalin-1,4-diolen nur durch kooperative Bewegungen verschiedener Molekïlteile erreicht wird, deren Auftreten innerhalb kurzer Zerfallszeiten wenig wahrscheinlich ist.

In Abb. 5 ist eine Zusammenstellung der FIK-Kurven für die transannulare 1,4-Eliminierung von $\mathrm{H}_{2} \mathrm{O}$ aus den Molekül-Ionen der verschiedenen Isomeren wiedergegeben. 
Auch in diesem Fall wird eine gute Übereinstimmung in der Lage der Intensitätsmaxima bei $3 \cdot 10^{-9} \mathrm{~s}$ beobachtet. Interessanterweise bilden Isomere mit diaxialer und mit diäquatorialer Anordnung der Hydroxygruppe zwei Kurvenscharen, wobei die Zerfallsrate der Molekül-Ionen mit diaxialen Hydroxygruppen im Maximum etwa doppelt so groß ist wie bei den anderen Isomeren.

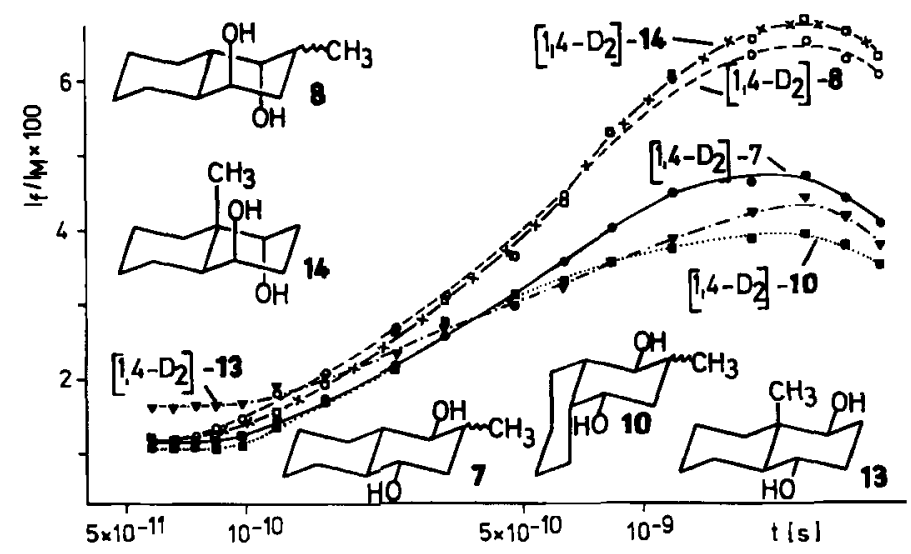

Abb. 5. Normalisierte Intensitätskurven für die Abspaltung von HDO durch transannulare 1,4-Eliminierung

Für diesen Effekt lassen sich zwei Gründe anführen, zwischen denen aufgrund der vorliegenden Messungen noch nicht entschieden werden kann.

1. Beim Ionisationsprozeß entstehen aus den Molekülen mit diaxialer und mit diäquatorialen Hydroxygruppen Molekül-Ionen mit unterschiedlicher Verteilung der Anregungsenergien, wobei die Isomeren mit diaxialen Hydroxygruppen mehr MolekülIonen mit geringer Anregungsenergie bilden. Dann stehen für den Zerfall durch die 1,4Eliminierung mit geringer Aktivierungsenergie mehr Molekül-Ionen zur Verfügung, während bei den Prozessen mit größerer Aktivierungsenergie kaum Unterschiede auftreten. Es wäre von Interesse, diese Möglichkeit an Hand der Photoelektronenspektren der stereoisomeren Decalin-1,4-diole zu prüfen.

2. Die transannulare 1,4-Eliminierung erfordert bei diaxialer Anordnung der Hydroxygruppen eine geringere Aktivierungsenergie als bei diäquatorialen Substituenten. Die Ursache dafür könnte eine größere Bildungsenthalpie der Isomeren mit diaxialen Hydroxygruppen aufgrund 1,3-diaxialer Wechselwirkungen sein. Die Wasserabspaltung durch 1,4-Eliminierung verläuft bei diaxialen und diäquatorialen Hydroxygruppen über geometrisch ähnliche Übergangszustände und liefert Ionen gleicher Konstitution. Die Aktivierungsenergie als Differenz der Bildungsenthalpien von Übergangs- und Ausgangszuständen sollte daher für das energiereichere Isomere kleiner sein. Dieser Effekt konnte durch Messungen der Auftrittspotentiale der $\left[\mathrm{M}-\mathrm{H}_{2} \mathrm{O}\right]^{+\cdot}$-Ionen in den EI-Massenspektren der Stereoisomeren bisher nicht nachgewiesen werden.

Neben den beiden zeitlich verzögerten Wasserabspaltungen durch 1,5- und 1,4-Eliminierungen, deren Ablauf mit Hilfe der spezifisch deuterierten Decalin-1,4-diol-Derivate nachgewiesen werden kann, weisen die FIK-Kurven in Abb. 2 für die Abspaltung von 
$\mathrm{H}_{2} \mathrm{O}$ aus den Molekül-Ionen von [1,4- $\left.\mathrm{D}_{2}\right]-10$ und die Abspaltung von $\mathrm{HDO}$ aus den Molekül-Ionen von 12-(OD) ${ }_{2}$ auf weitere zeitlich verzögerte Eliminierungsprozesse hin. Die normalisierten Intensitätskurven zeigen bei kurzen Zerfallszeiten mit der hohen Anfangsintensität und dem nachfolgenden steilen Abfall den typischen Verlauf der schnellen 1,2-Eliminierung; die Intensität nimmt bei längeren Zerfallszeiten aber wieder zu und erreicht für beide Verbindungen bei ca. $3 \cdot 10^{-9} \mathrm{~s}$ ein Maximum. Dieses abweichende Verhalten wird bei einem Vergleich mit der entsprechenden FIK-Kurve für das dritte Stereoisomere 11 des 2-Methyl-cis-decalin-1,4-diols besonders deutlich (Abb. 6), da diese Kurve mit dem gleichförmigen Absinken der Ionenintensität dem Verlauf der FIKKurven bei den trans-Decalin-1,4-derivaten (Abb. 3) entspricht.

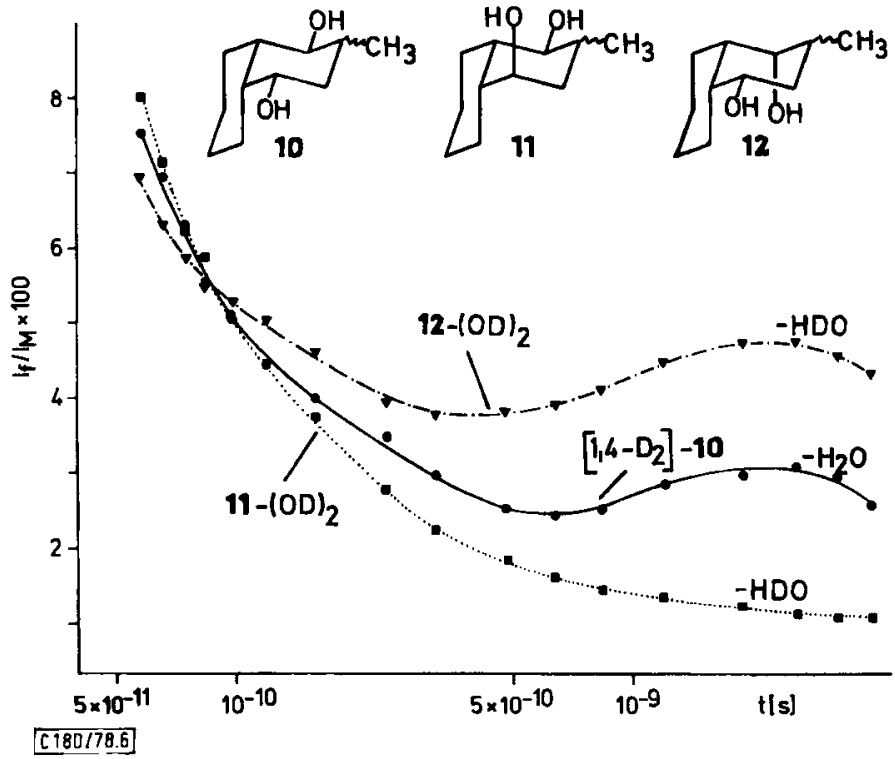

Abb. 6. Normalisierte Intensitätskurven für die Abspaltung von $\mathrm{H}_{2} \mathrm{O}$ bzw. $\mathrm{HDO}$ aus den Molekül-Ionen deuterierter 2-Methyl-cis-decalin-1,4-diole

Es fällt auf, daß dieser Effekt nur bei solchen Derivaten des cis-Decalin-1,4-diols beobachtet wird, bei denen Konformationen mit kurzen Abständen zwischen mindestens einer der Hydroxygruppen und den H-Atomen am anderen Ring möglich sind: Bei 12, das derartige Konformationen in doppelter Anzahl besitzt wie 10 (Schema 5), tritt dieser Eliminierungsprozeß besonders häufig auf, bei 11 ohne eine derartige Konformation wird er dagegen nicht gefunden.

Diese Wasserabspaltung erreicht ihre maximale Rate bei vergleichbaren Zerfallszeiten wie die transannulare 1,4-Eliminierung, so daß ihr Übergangszustand nur durch eine ähnlich komplizierte Molekülbewegung erreicht werden kann. Tatsächlich zeigen Molekül-Modelle, daß nach der Umwandlung des unsubstituierten Ringes von 10 und 12 in eine Bootkonformation (Schema 5) zwischen einer axialen Hydroxygruppe und einem H-Atom in Position 6 ein extrem kurzer Abstand von ca. $0.5 \AA$ eingestellt werden kann. Im Gegensatz zur transannularen 1,4-Eliminierung ist eine derartige Wasserabspaltung 
nicht durch eine schwache $\mathrm{C}-\mathrm{H}$-Bindung energetisch begünstigt. In der reaktiven Konformation dieser Abspaltung kann jedoch der kleinste in einem Decalin-1,4-diol mögliche Abstand von $\mathrm{H}$ - und O-Atom erreicht werden, so daß die H-Übertragung kaum zusätzliche Schwingungsanregung erfordert. Dies deutet auf eine geringe Aktivierungsenergie der Eliminierung hin, so daß der Proze $\beta$ bei den langen Zerfallszeiten wenig angeregter Molekül-Ionen noch mit der energetisch günstigen 1,4-Eliminierung in 10 konkurrieren kann.
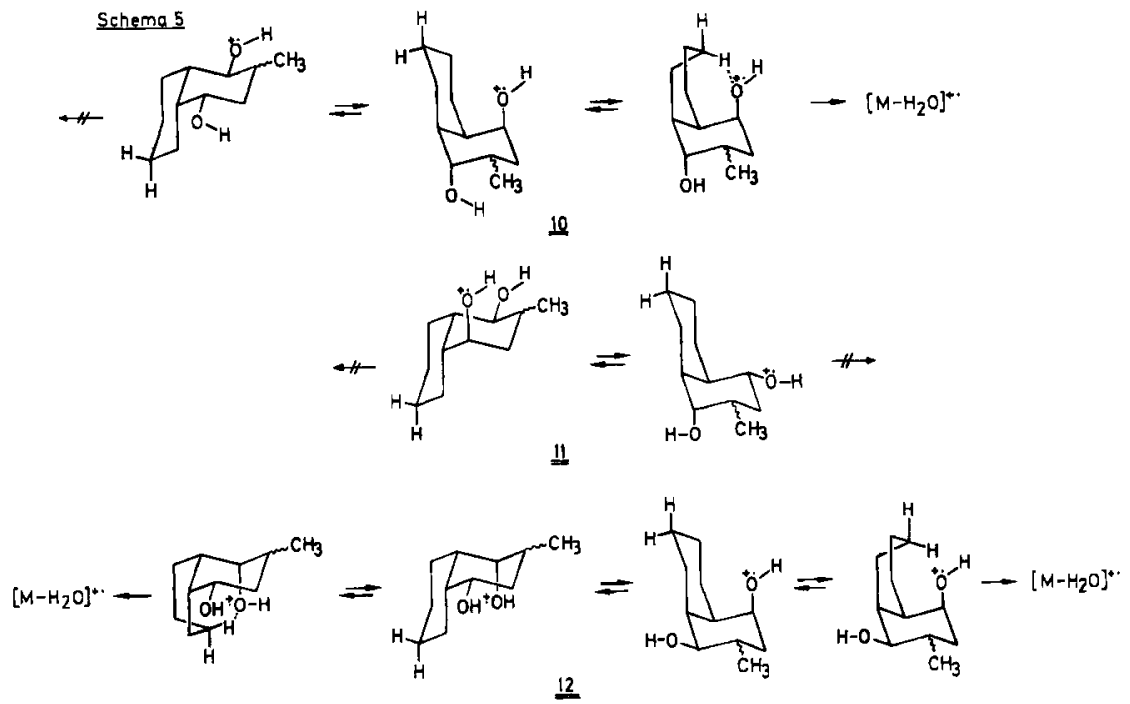

Die vorliegenden Ergebnisse zeigen sehr deutlich den Vorteil der FIK-Methode gegenüber den üblichen FI- und EI-Massenspektren. So unterscheiden sich die FI-Massenspektren der stereoisomeren Decalin-1,4-diole praktisch nicht, weil bei den kurzen Reaktionszeiten die Ionen $\left[\mathrm{M}-\mathrm{H}_{2} \mathrm{O}\right]^{+\cdot}$ nur durch die in allen Isomeren gleichermaßen mögliche 1,2-Eliminierung entstehen, die von der Stereochemie der Diole gesteuerten Prozesse dagegen erst bei längeren Reaktionszeiten eintreten und von den FI-Massenspektren nicht erfaßt werden. In den El-Massenspektren werden zwar auch Ionen registriert, die bis $\mathrm{zu}$ Reaktionszeiten von ca. $10^{-6} \mathrm{~s}$ gebildet werden, jedoch wird bei der Messung über diesen Zeitraum integriert. Zusammen mit der häufigen Bildung energiereicher Molekül-Ionen bei der Elektronenstoß-Ionisation kann dies zu einer Nivellierung sterischer Effekte auf die Ionenintensitäten führen. Demgegenüber liefert die Analyse der FIK-Kurven mehr Informationen über den Ablauf der Eliminierungsreaktionen und ihre Abhängigkeit von der Molekülgeometrie. Man gewinnt dabei den Eindruck, daß die Mechanismen massenspektrometrischer Fragmentierungen mehr als bisher vermutet von intramolekularen Orientierungseffekten beeinflußt werden. Neben strukturanalytisch verwertbaren Angaben für die relative Orientierung ausgezeichneter Atomgruppen in organischen Molekülen kann die FIK-Untersuchung massenspektrometrischer Fragmentierungen als Prototypen schneller unimolekularer Reaktionen angeregter Teilchen daher auch Hinweise liefern für den Einfluß der molekularen Beweglichkeit auf schnelle Reaktionen. 
Dem Minister für Forschung und Wissenschaft des Landes Nordrhein-Westfalen und dem Fonds der Chemischen Industrie danken wir für die finanzielle Unterstützung dieser Arbeit.

\section{Experimenteller Teil}

Die Schmelzpunkte sind unkorrigiert. - IR-Spektren: Perkin-Elmer 377 Spektrophotometer, KBr-PreBlinge. - ${ }^{1} H-N M R$ - und ${ }^{13} C-N M R$-Spektren: Gerät Bruker WP 80 (FT-Gerät), Lösungsmittel $\left[\mathrm{D}_{6}\right]$ Aceton, bei $8\left[\mathrm{D}_{6}\right] \mathrm{DMSO}$. - Massenspektren: Doppelfokussierendes Massenspektrometer MAT 311 A (Varian MAT, Bremen) und EI/FI-Kombinations-Ionenquelle Varian MAT. Betriebsbedingungen: Ionenquellentemperatur $180^{\circ} \mathrm{C}$, Probenzuführung durch Direkteinlaßsystem, Druck in der Ionenquelle während der Messung ca. $5 \cdot 10^{-6}$ Torr. Potentialdifferenz Feldemitter-Gegenelektrode (Saugblende) bei normalem FI-Betrieb ca. $7.5 \mathrm{kV}$. Hochspannung des SEV-Systems $2.2 \mathrm{kV}$, Bandbreite des Gleichstromverstärkers BDF $100 \mathrm{~Hz}$. Die FI-Massenspektren (und FIK-Messungen) wurden unter Verwendung konditionierter ${ }^{2)}$ Drahtemitter (s. unten) aus W-Fäden ( $5 \mu \mathrm{m}$ Radius) durchgeführt. Vor den Messungen wurden die Emitter durch Ausheizen mit einem Heizstrom von $50 \mathrm{~mA}$ für 1 - 2 min gereinigt. Während der Messungen wurde zur Vermeidung von Kondensationen auf der Emitter-Oberfläche mit einem Strom von ca. $10 \mathrm{~mA}$ geheizt.

Aktivierung der Emitterfälen: Die Fäden wurden in einer Aktivierungszelle (Varian MAT) konditioniert, die eine weitgehend reproduzierbare Aktivierung ermöglicht. Die Aktivierung erfolgt in einer Benzonitril-Atmosphäre bei einem Druck von ca. $8 \cdot 10^{-3}$ Torr. Die Fäden befinden sich auf einem Potential von $+10 \mathrm{kV}$ gegenüber einer $2 \mathrm{~mm}$ entfernten geerdeten Gegenelektrode. Sie werden durch einen Heizstrom von $38 \mathrm{~mA}$ erwärmt. Die Hochspannung wurde stets bei Erreichen eines Emissionsstromes von $2 \mu \mathrm{A} u \mathrm{~m} 1 \mathrm{kV}$ gesenkt bis zum Endwert von $6 \mathrm{kV}$. Die Aktivierung wird beendet bei einem Emissionsstrom von $2.5 \mu \mathrm{A}$ bei $6 \mathrm{kV}$ Potentialdifferenz. Gesamtdauer der Aktivierung 4-5 h. Der Radius des aktivierten Emitters wurde zu $15 \pm 0.5 \mu \mathrm{m}$ bestimmt.

Feldionenkinetische Untersuchungen: Die kinetische Energie der Fragment-Ionen wurde durch Veränderung des Emitterpotentials (gegen Erdpotential) bestimmt. Die Potentialdifferenz für die Feldionisation wird beim Massenspektrometer MAT $311 \mathrm{~A}$ durch Anlegen eines positiven Potentials an den Emitter und eines negativen Potentials an die Gegenelektrode (Saugblende) erzeugt. Der Abstand Emitter - Gegenelektrode wurde zu $1.35 \pm 0.01 \mathrm{~mm}$ bestimmt.

Das (negative) Potential der Saugblende wird von einem Hochspannungsgerät „Brandenburg“ geliefert und kann an dem Gerät abgelesen werden. Für jede Substanz wurden nacheinander Messungen bei vier verschiedenen konstanten Saugblendenspannungen von $-6.0,-4.5,-3.5$ und $-2.0 \mathrm{kV}$ durchgeführt.

Das (positive) Potential des Emitters kann am MAT $311 \mathrm{~A}$ zwischen +1 und $+3 \mathrm{kV}$ kontinuierlich eingestellt werden und wurde mit einem Digital-Voltmeter "Data Precision 2540 A 1" gemessen.

Meßvorgang und Auswertung: Wührend der FIK-Messungen wurden die Fokussierlinsen der FI-Ionenquelle geerdet. Das Massenspektrometer wurde bei vorgewählter Saugblendenspannung und einer Emitter-Spannung von $+2 \mathrm{kV}$ auf die Masse der $\left[\mathrm{M}-\mathrm{H}_{2} \mathrm{O}\right]^{+} \cdot$-Ionen einjustiert und der lonenstrom mit einem Kompensationsschreiber Kompensograph (Fa. Siemens) registriert. Anschließend wurde das Emitterpotential in definierten Schritten von $20 \mathrm{~V}$ bis zu einem Grenzwert von $3 \mathrm{kV}$ erhöht und die Ionenströme $\left[\mathrm{M}-\mathrm{H}_{2} \mathrm{O}\right]^{+\cdot}$ sowie der Total-lonenstrom bei jeder Emitterspannung registriert. Alle Messungen wurden fünfmal wiederholt und die Meßwerte gemittelt. Die Mittelwerte werden entsprechend der Änderung des Total-Ionenstroms korrigiert und auf die Intensität der Molekül-Ionen beim Ausgangswert der Emitterspannung normiert. 
Mit Hilfe der Formel ${ }^{13 a, 25)}$

$$
U_{x}=\frac{\Delta U}{1-\frac{m_{1}}{m_{2}}}+U_{0}
$$

$U_{\mathrm{x}}=$ Potentialdifferenz zwischen Zerfallsort und Kathode

$U_{0}=$ Anfangspotentialdifferenz zwischen Emitter und Kathode

$\Delta U=$ Änderung des Emitterpotentials

$m_{1}=$ absolute Masse des Mutter-Ions

$m_{2}=$ absolute Masse des Tochter-Ions

wird eine Tabelle aufgestellt, die den Ionenstrom beim jeweiligen Wert von $U_{\mathrm{x}}$ angibt. Alle Werte von $U_{\mathrm{x}}$, die aus einem Zerfallsort mit negativem Potential berechnet werden, wurden nicht verwendet, da es zwei Orte mit diesen Potentialen gibt.

Anschließend wird eine Tabelle Ionenstromintensität gegen die Zeit $t$ mit folgender Formel errechnet ${ }^{2)}$

$$
\begin{gathered}
t=\frac{2 r \sqrt{\ln \frac{R}{r}}}{\sqrt{\frac{2 e\left(U_{0}+\Delta U\right)}{m_{1}}}} \int_{0}^{y_{0}} e^{y^{2}} \mathrm{~d} y \\
y_{0}=\sqrt{\frac{U_{0}+\Delta U-U_{x}}{U_{0}+\Delta U}} \ln \frac{R}{r} \\
r=\text { Emitterradius }=1.5 \cdot 10^{-5} \mathrm{~m}\left( \pm 0.05 \cdot 10^{-5} \mathrm{~m}\right) \\
R=\text { Abstand-Emitter-Kathode }=1.35 \cdot 10^{-3} \mathrm{~m} \\
e=\text { Elementarladung }=1.6021 \cdot 10^{-19} \mathrm{C}
\end{gathered}
$$$$
R=\text { Abstand-Emitter-Kathode }=1.35 \cdot 10^{-3} \mathrm{~m}
$$

Das Integral wurde mittels numerischer Integration tabelliert.

Da die Messungen nacheinander mit vier verschiedenen Potentialdifferenzen $U_{0}$ durchgeführt wurden (s. oben), erhält man vier Ionenstrom-Zeit-Kurven mit etwas verschobenem Zeitfenster: längste erfaßbare Zeit bei $U_{0}=8 \mathrm{kV} \cong 1.2 \cdot 10^{-9} \mathrm{~s}$; bei $U_{0}=4 \mathrm{kV} \cong 4 \cdot 10^{-9} \mathrm{~s}$.

Die Ionenstromintensitäten für jedes $U_{0}$ werden durch einen Faktor korrigicrt, so daß sämtliche Ionenstromkurven im gemeinsamen Zeitintervall identisch sind mit der Kurve, die für den größten Wert von $U_{0}$ aufgenommen wurde.

Die Reproduzierbarkeit der FIK-Kurven ist gut. Die mittlere Abweichung der Ionenstromintensitäten beträgt bei fünf Messungen für $U_{0}=8 \mathrm{kV}$ etwa $4 \%$, für $U_{0}=4 \mathrm{kV}$ etwa $7 \%$.

Darstellung der Decalin-1,4-diole: Die stereoisomeren Diole wurden nach Hückel und Kraus ${ }^{9}$ ) dargestellt:

2-Methyl-trans-decalin-1,4-diole (7-9): Die Lösung von $5.0 \mathrm{~g}$ (27.8 mmol) 2-Methyl-transdecalin-1,4-dion ${ }^{26}$ in $50 \mathrm{ml}$ Ethanol wurde während $1 \mathrm{~h}$ zu einer Suspension von $1.1 \mathrm{~g}(29.1 \mathrm{mmol})$ Natriumborhydrid in $50 \mathrm{ml}$ Ethanol getropft. Nach Hydrolyse mit verd. Schwefelsäure wurde das Ethanol abgezogen und der Rückstand in Ether aufgenommen. Es wurde zweimal mit wenig gesättigter Natriumchlorid-Lösung gewaschen, dann ausgeethert, getrocknet und der Ether abgezogen. Ausb. $3.1 \mathrm{~g} \mathrm{(61 \% )} \mathrm{Gemisch} \mathrm{stereoisomerer} \mathrm{Diole.} \mathrm{Säulenchromatographie} \mathrm{(Kieselgel,}$ Benzol/Aceton 2:1) lieferte $1.67 \mathrm{~g}(54 \%)$ 9, Schmp. $113^{\circ} \mathrm{C}$, IR (KBr): 959, 998 (a-C - O), 1034, $1052 \mathrm{~cm}^{-1}$ (e-C-O); $0.62 \mathrm{~g}(20 \%)$ 7, Schmp. $147^{\circ} \mathrm{C}$, IR (KBr): $1019,1038 \mathrm{~cm}^{-1}(\mathrm{e}-\mathrm{C}-\mathrm{O})$, und $0.81 \mathrm{~g}(26 \%) 8$, Schmp. $156^{\circ} \mathrm{C}, \mathrm{IR}(\mathrm{KBr}): 979,986 \mathrm{~cm}^{-1}$ (a-C $\left.-\mathrm{O}\right)$.

$$
\begin{array}{rr}
\mathrm{C}_{11} \mathrm{H}_{20} \mathrm{O}_{2} \text { (184.3) } & \text { Ber. C } 71.70 \text { H } 10.94 \\
\text { 7: } & \text { Gef. C } 71.96 \text { H } 10.84 \\
\text { 8: } & \text { Gef. C } 71.73 \text { H } 10.69 \\
\text { 9: } & \text { Gef. C } 71.28 \text { H } 10.71
\end{array}
$$


2-Methyl-cis-decalin-1,4-diole (10 - 12): Die Lösung von $7.0 \mathrm{~g}$ (38.9 mmol) 2-Methyl-cis-decalin1,4-dion ${ }^{26)}$ in $100 \mathrm{ml}$ trockenem Ether wurde langsam unter Rühren zu $3 \mathrm{~g} \mathrm{(78.9} \mathrm{mmol)} \mathrm{Lithium-}$ aluminiumhydrid in $100 \mathrm{ml}$ Ether getropft. Nach Hydrolyse mit 10proz. Schwefelsäure wurde mit Ether extrahiert. Es wurden $6.1 \mathrm{~g}(85 \%)$ Gemisch stereoisomerer Diole erhalten. Säulenchromatographische Trennung (Aluminiumoxid, neutral, Essigester/Petrolether $\left.\left(60-70^{\circ} \mathrm{C}\right)(2: 1)\right)$ lieferte $5.06 \mathrm{~g} \mathrm{(83 \% )} \mathrm{12,} \mathrm{Schmp.} 39^{\circ} \mathrm{C}$, IR (KBr): $989(\mathrm{a}-\mathrm{C}-\mathrm{O}), 1033 \mathrm{~cm}^{-1}(\mathrm{c}-\mathrm{C}-\mathrm{O}) ; 0.73 \mathrm{~g} \mathrm{(12 \% )} 10$, Schmp. $176^{\circ} \mathrm{C}$, IR (KBr): 1028 (e-C-O), $1044 \mathrm{~cm}^{-1}$ (e-C-O), und $0.31 \mathrm{~g} \mathrm{(5 \% )} \mathrm{11,} \mathrm{Schmp.} 138^{\circ} \mathrm{C}$, IR (KBr): 987 (a-C-O), $1028 \mathrm{~cm}^{-1}$ (e-C-O).

$$
\begin{array}{rll}
\mathrm{C}_{11} \mathrm{H}_{20} \mathrm{O}_{2}(184.3) & \text { Ber. C } 71.70 \text { H } 10.94 \\
10: & \text { Gef. C } 71.83 \text { H } 10.74 \\
11: & \text { Gef. C } 71.89 \text { H } 10.73 \\
\text { 12: } & \text { Gef. C } 71.66 \text { H } 10.78
\end{array}
$$

9-Methyl-trans-decalin-1,4-diole (13-15): Die Lösung von $7.0 \mathrm{~g}$ (38.9 mmol) 9-Methyl-transdecalin-1,4-dion ${ }^{27)}$ in $150 \mathrm{ml}$ trockenem Ether wurde langsam unter Rühren zu $4.0 \mathrm{~g}(105.3 \mathrm{mmol})$ $\mathrm{LiAlH}_{4}$ in $50 \mathrm{ml}$ Ether getropft. Nach Hydrolyse mit 10 proz. Schwefelsäure wurde mit Ether extrahiert. Es wurden $6.67 \mathrm{~g}$ (93\%) Gemisch stereoisomerer Diole erhalten. Säulenchromatographie (Kieselgel, Toluol/Aceton 2:1) ergab $0.73 \mathrm{~g}$ (11\%) 13, Schmp. $121^{\circ} \mathrm{C}, \mathrm{IR}(\mathrm{KBr})$ : 1019 , $1051 \mathrm{~cm}^{-1}$ (e-C-O), $3.07 \mathrm{~g}(46 \%)$ 15, Schmp. $154^{\circ} \mathrm{C}$, IR (K Br): 981 (a-C-O), $1034 \mathrm{~cm}^{-1}(\mathrm{e}-\mathrm{C}-\mathrm{O})$, und $2.87 \mathrm{~g}(43 \%) 14$, Schmp. $163^{\circ} \mathrm{C}$, IR (KBr): $973,1000 \mathrm{~cm}^{-1}$ (a-C-O).

$$
\begin{aligned}
\mathrm{C}_{11} \mathrm{H}_{20} \mathrm{O}_{2}(184.3) & \text { Ber. C } 71.70 \text { H } 10.94 \\
13: & \text { Gef. C } 71.75 \text { H } 10.84 \\
\text { 14: } & \text { Gef. C } 71.61 \text { H } 10.79 \\
\text { 15: } & \text { Gef. C } 71.38 \text { H } 10.76
\end{aligned}
$$

Die Zuordnung der Stereoisomeren erfolgte anhand der chemischen Verschiebung und der Halbwertsbreite der Signale der Protonen am Carbinol-C-Atom in den ${ }^{1} \mathrm{H}-\mathrm{NMR}$-Spektren entsprechend der von Hückel und Kraus ${ }^{28)}$ durchgeführten Zuordnung der stereoisomeren Decalin-1,4-diole ${ }^{28}$.

Tab.: Chemische Verschiebung und Halbwertsbreite der Protonen am Carbinol-C-Atom in den ${ }^{1}$ H-NMR-Spektren

\begin{tabular}{rll}
\hline Isomere & \multicolumn{1}{c}{$\tau$} & Halbwertsbreite $[\mathrm{Hz}]$ \\
\hline $\mathbf{7}$ & 3.10 & $8.5 \pm 0.5$ \\
$\mathbf{8}$ & 3.57 & $4.0 \pm 0.5$ \\
$\mathbf{9}$ & $3.42 ; 3.67$ & $8.0 \pm 0.5 ; 11.0+1.0$ \\
$\mathbf{1 0}$ & 3.26 & $14.5 \pm 1.0$ \\
$\mathbf{1 1}$ & 3.12 & $6.5 \pm 0.5$ \\
$\mathbf{1 2}$ & 3.37 & $6.0 \pm 0.5$ \\
$\mathbf{1 3}$ & 3.25 & $7.5 \pm 0.5$ \\
$\mathbf{1 4}$ & 3.73 & $3.5 \pm 0.5$ \\
$\mathbf{1 5}$ & $3.26 ; 3.42$ & $4.0 \pm 0.5 ; 9.0 \pm 0.5$ \\
\hline
\end{tabular}

Die Zuordnung der Isomeren 11 und 12 wurde zusätzlich wie bei den Isomeren 5 und 6 durchgeführt ${ }^{9)}$ : 11 gibt wie 5 ein stabiles Toluolsulfonat, Schmp. $161^{\circ} \mathrm{C}$, während sich das Toluolsulfonat von 12 wie bei 6 rasch zersetzt.

Die Konfiguration von 15 wurde aus der chemischen Verschiebung der Carbinol-C-Atome in den ${ }^{13} \mathrm{C}-\mathrm{NMR}$-Spektren durch Vergleich mit den ${ }^{13} \mathrm{C}-\mathrm{NMR}$-Spektren der 9-Methyl-transdecalin-1-ole bzw. -4-ole bestimmt ${ }^{29)}$. Auf diese Weise wird bei 7-12 nur die Konfiguration an 
C-1 und C-4 bestimmt, nicht jedoch die an C-2. Nach Aussage der ${ }^{13} \mathrm{C}-$ NMR-Spektren sind alle Verbindungen ein Gemisch der Konfigurationsisomeren an C-2 ${ }^{29}$.

Die 1,4-C-deuterierten Diole wurden wie die undeuterierten Verbindungen dargestellt, wobei $\left[\mathrm{D}_{4}\right]$ Lithiumaluminiumhydrid bzw. $\left[\mathrm{D}_{4}\right]$ Natriumborhydrid als Reduktionsmittel verwendet wurden.

D-Gehalt (massenspektrometrisch bestimmt):

$$
\begin{array}{lll}
{\left[1,4-D_{2}\right]-7:} & 4 \% D_{1}, 96 \% D_{2} & {\left[1,4-D_{2}\right]-11: 5 \% D_{1}, 95 \% D_{2}} \\
{\left[1,4-D_{2}\right]-8:} & 4 \% D_{1}, 96 \% D_{2} & {\left[1,4-D_{2}\right]-12: 4 \% D_{1}, 96 \% D_{2}} \\
{\left[1,4-D_{2}\right]-9:} & 5 \% D_{1}, 95 \% D_{2} & {\left[1,4-D_{2}\right]-13: 2 \% D_{1}, 98 \% D_{2}} \\
{\left[1,4-D_{2}\right]-10: 5 \% D_{1}, 95 \% D_{2}} & {\left[1,4-D_{2}\right]-14: 2 \% D_{1}, 98 \% D_{2}}
\end{array}
$$

Die 1,4-di-O-deuterierten Diole wurden durch Deuteriumaustausch erhalten: Die Lösung des jeweiligen Diols in möglichst wenig absol. Tetrahydrofuran wurde $1 \mathrm{~h}$ mit dem 1000 fachen Uberschuß an $\mathrm{D}_{2} \mathrm{O}$ gerührt. Anschließend wurden das Lösungsmittel und $\mathrm{D}_{2} \mathrm{O}$ abgezogen. D-Gehalt (massenspektrometrisch bestimmt):

$$
\begin{array}{rl}
7-(O D)_{2}: 6 \% D_{1}, 94 \% D_{2} & 11-(O D)_{2}: 1 \% D_{0}, 8 \% D_{1}, 91 \% D_{2} \\
8-(O D)_{2}: 6 \% D_{1}, 94 \% D_{2} & 12-(O D)_{2}: 7 \% D_{1}, 93 \% D_{2} \\
9-(O D)_{2}: 7 \% D_{1}, 93 \% D_{2} & 13-(O D)_{2}: 6 \% D_{1}, 94 \% D_{2} \\
10-(O D)_{2}: 6 \% D_{1}, 94 \% D_{2} & 14-(O D)_{2}: 5 \% D_{1}, 95 \% D_{2}
\end{array}
$$

Das $\left[2,2,3,3,9,10-D_{6}\right]$-Derivat des Decalin-1,4-diols 1 stand aus früheren Untersuchungen zur Verfügung ${ }^{7}$.

\section{Literatur}

1) Mechanismus massenspektrometrischer Fragmentierungsreaktionen, XXI; XX. Mitteil.: H. Fr. Grützmacher und G. Lange, Chem. Ber. 111, 1962 (1978).

${ }^{21}$ H.D. Beckey,Principles of Field lonization and Field Desorption Mass Spectrometry, Pergamon Press, Oxford 1977.

3) P. J. Derrick und A. L. Burlingame, Acc. Chem. Res. 7, 328 (1974).

4) E. L. Eliel, Stereochemie der Kohlenstoffverbindungen, Verlag Chemie, Weinheim/Bergstr. 1966.

5) C. G. Mac Donald, J. S. Shannon und G. Sugowdz, Tetrahedron Lett. 1963, 807.

6) H. Fr. Grüt zmacher Suom. Kemistil. 46, 50 (1973).

7) H. Fr. Grützmacher und K. H. Fechner, Org. Mass Spectrom. 7, 573 (1973).

${ }^{8)}$ H. Fr. Grützmacher und R. Asche, Chem. Ber. 108, 2080 (1975).

9) W. Hückel und W. Kraus, Chem. Ber. 95, 233 (1962).

${ }^{10)}$ H. Fr. Grützmacher, G. Tolkien und R. Heller, Advances in Mass Spectrometry(Herausg. N. R. Daly) Vol. 7 B, S. 1176, Heyden and Son Ltd., London 1978.

II) Zit. 2), Kap. 4.

${ }^{12)}$ K. Levsen und H. D. Beckey, Int. J. Mass Spectrom. Ion Phys. 15, 353 (1974).

13) 13s) A. M. Falick, P. J. Derrick und A. L. Burlingame, Int. J. Mass Spectrom. Ion Phys. 12, 101 (1973). - 13b) P. J. Derrick, R. P. Morgan, J. T. Hill und M. A. Baldwin, ebenda 18, 393 (1975). i 3c) D. G. Patterson, R. B. Scott und P. Brown, Org. Mass Spectrom. 12, 395 (1977).

14) Zit. 2), S. 179.

15) J. D. Beckey, K. Levsen und P. J. Derrick, Org. Mass Spectrom. 11, 835 (1976).

16) H. M. Rosenstock, M. B. Wallenstein, A. L. Wahrhaftig und H. Eyring, Proc. Nat. Acad. Sci., U. S. 38, 667 (1952). 
${ }^{17)}$ G. Treuschert und H. D. Beckey, Int. J. Mass Spectrom. Ion Phys. 7, 97 (1971).

18) O. K. Rice, J. Phys. Chem. 65, 1588 (1961).

19) Zit. 2), S. $200 \mathrm{ff}$.

${ }^{20)}$ K. Levsen und H. D. Beckey, Int. J. Mass Spectrom. Ion Phys. 7, 341 (1971).

21) P. J. Derrick, A. M. Falick und A. L. Burlingame, J. Am. Chem. Soc. 95, 437 (1973).

22) P. J. Derrick, J. L. Holmes und R. P. Morgan, J. Am. Chem. Soc. 97,4936 (1975).

${ }^{23)}$ M. M. Green, R. J. Cook, J. M. Schwab und R. B. Roy, J. Am. Chem. Soc. 92,3076 (1970).

${ }^{24)}$ R. H. Martin, F. W. Lampe und R. W. Taft, J. Am. Chem. Soc. 88, 1353 (1966).

25) A. M. Falick, Int. J. Mass Spectrom. Ion Phys. 14, 313 (1974).

26) W. S. Johnson, D. S. Allen jr., R. R. Hindersinn, G. N. Sausen und R. Pappo, J. Am. Chem. Soc. 84, 2192 (1962).

27) V. Georgian und L. L. Skaletzky, J. Org. Chem. 29, 51 (1964).

28) W. Hückel und W. Kraus, Liebigs Ann. Chem. 683, 75 (1965).

${ }^{29)}$ G. Tolkien, Dissertation, Univ. Bielefeld 1978. 